\title{
Incidental Heterotopic Mesenteric Ossifications
}

\author{
Beshoy Nashed ${ }^{\mathrm{a}, \mathrm{b}}$, Wesley High ${ }^{\mathrm{a}, \mathrm{b}}$, Syed Zubair Tahira, Christopher Quijano ${ }^{\mathrm{a}}$, \\ Shea Jones ${ }^{\text {a }}$
}

\begin{abstract}
Very few cases of heterotopic mesenteric ossification (HMO) have been reported in literature. This benign growth of bony tissue in the mesentery of the small or large bowel appears to follow repetitive or severe abdominal injuries. This is a case of a 24 -year-old male who developed HMO after multiple surgeries following complications of a diverticular abscess.
\end{abstract}

Keywords: Mesenteric heterotopic ossifications; Mesenteric bones; Bowel obstruction; Exploratory laparotomy; Multiple abdominal surgeries; Abdominal trauma; Bones

\section{Introduction}

Heterotopic ossification is described as the formation of bony tissue that does not normally ossify [1]. The presence of osteoblasts within the calcified tissue and the formation of lamellar bone make heterotopic ossification a distinct entity from other forms of ectopic calcifications. Heterotopic ossification is a relatively common complication in orthopedic surgery, especially following total hip arthoplasty; it remains a rare event in abdominal surgery. To date, only 44 cases have been described in the literature. The finding of heterotopic ossification following abdominal surgery was first reported by Hansen et al [2], and the term heterotopic mesenteric ossification (HMO) is attributed to Wilson et al [3] to describe a series of patients with heterotopic ossification of the mesentery following abdominal surgery.

The pathology of HMO remains elusive; however, it is nearly universally associated with abdominal trauma or surgery. HMO commonly presents as either a small bowel ob-

\footnotetext{
Manuscript accepted for publication July 23, 2015

${ }^{a}$ Mountain Vista Medical Center, General Surgery, Mesa, AZ, USA

${ }^{b}$ Corresponding Author: Beshoy Nashed, Mountain Vista Medical Center, $1301 \mathrm{~S}$. Crismon Road, Mesa, AZ 85209, USA. Email: banashed@iasishealthcare.com; Wesley High, Mountain Vista Medical Center, 1301 S. Crismon Road, Mesa, AZ 85209, USA. Email: whigh@iasishealthcare.com
}

doi: http://dx.doi.org/10.14740/jes273w struction or an enterocutaneous fistula in patients with a history of abdominal trauma and or surgery. Presented here is a review of the literature and an additional case of HMO in a 24-year-old male following a sigmoid colon resection complicated by an anastomotic leak.

\section{Case Report}

This is a 24-year-old obese Hispanic male who presented with diverticulitis with pericolonic abscess. The non-operative management failed and the patient underwent an uneventful sigmoid resection. He subsequently developed an anastomotic leak and returned to the OR for a transverse colostomy. He was discharged home after a XenMatrix porcine graft was used to close the fascia of the abdomen. The patient recovered for 4 months uneventfully with $50 \%$ closure of his abdominal wound. At that time, he presented back with two draining enterocutaneous fistulas eroding through the XenMatrix. He was taken back to the OR for takedown of fistulas and mesh removal. During this operation, it was discovered that he had three very sharp and large fragments of bone, all very different in shape, scattered throughout his small bowel mesentery with the largest measuring $7.5 \mathrm{~cm}$ in length (Fig. 1). These fragments were all confirmed by pathology to be ossified bone tissue without malignancy (Fig. 2). Interestingly enough, the

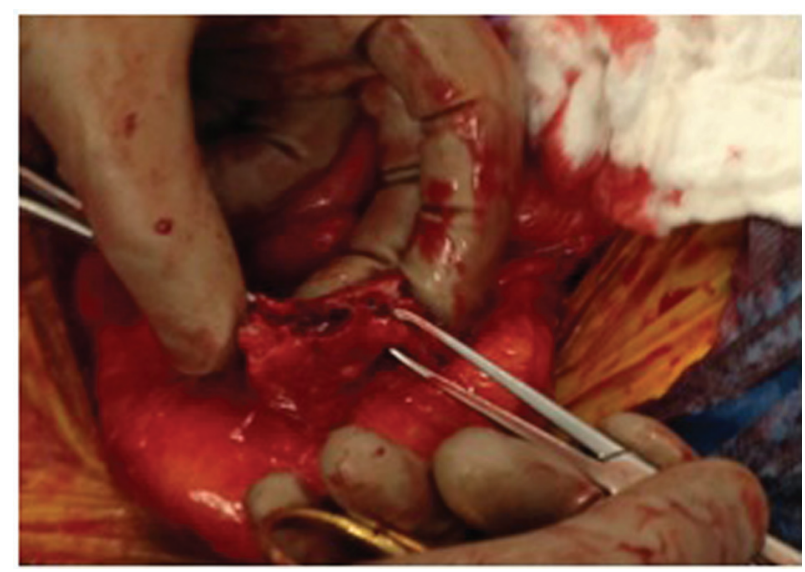

Figure 1. Ossified tissue, found in its most common location, in the root of the mesentery. 


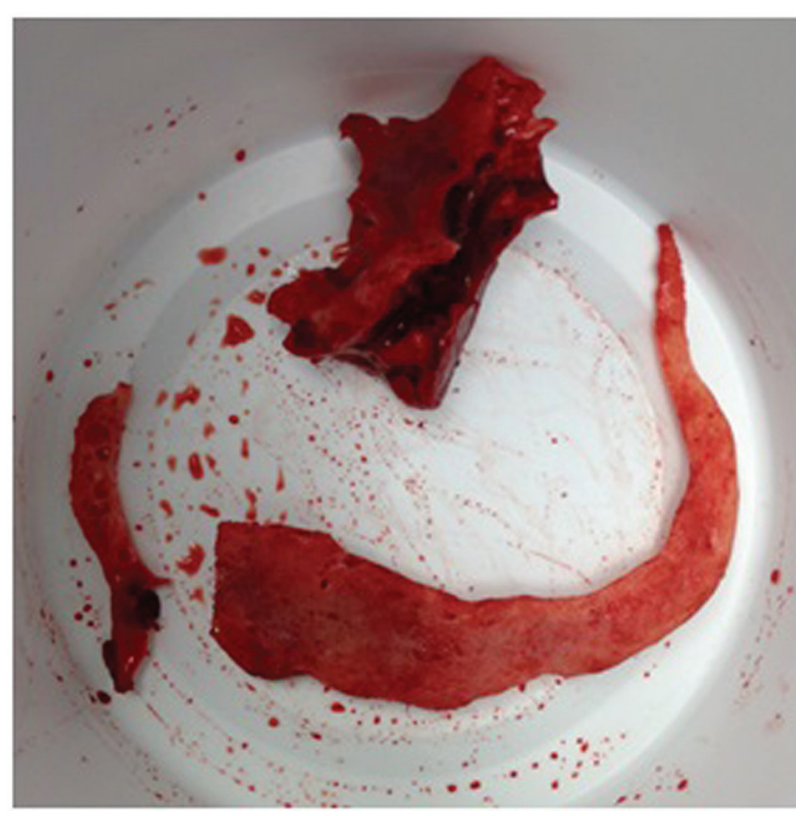

Figure 2. Removed specimens of heterotopic mesenteric ossification.

bone fragments did not appear to be in proximity of the enterocutaneous fistulas.

\section{Discussion}

Heterotopic ossification is a generic term used to describe bone formation in soft tissue and when it occurs in the mesentery of omentum is referred to as HMO. It occurs in the setting of abdominal catastrophes that require multiple abdominal surgeries to manage. The exact etiology remains unclear; however, several mechanisms have been proposed.

The theory behind the mechanism of the HMO has evolved over the years and remains to be definitively determined. The original theory revolved around the notion of seeding of osteogenic cells that were disrupted from either the periosteum or perichondrium [4]. However, in our case and others in the literature, the absence of periosteum or perichondrium disruption and with formation of HMO, the seeding theory fails to explain the development of HMO. Therefore, additional theories have been developed and the prevailing theory proposes a metaplastic response that involves four factors that contribute to the development of HMO [5]. First, there must be an initial insult, such as trauma, surgical wounds or sepsis, followed by the release of signals from the site of injury by the initially injured cells or by inflammatory cells. It is thought that bone morphogenic proteins (BMPs) may provide these signals [6]. BMPs are multifunctional cytokines that are part of the transforming growth factor- $\beta$ family. They have been shown to induce heterotopic cartilage and bone with bone marrow formation in skeletal muscles [7]. Further supporting the metaplastic theory are the histologic findings commonly associated with HMO.

The histologic characteristics commonly found in HMO include fibrous septa entrapping adipose tissue, nerves and
Table 1. Summary of Reported Cases of Heterotopic Mesenteric Ossification

\begin{tabular}{ll}
\hline Total cases & $\mathrm{n}=44$ \\
Sex & \\
\multicolumn{1}{l}{ Male } & 42 \\
Female & 2 \\
Location & \\
$\quad$ Mesentery & 39 \\
Omentum & 6 \\
Abdominal wall & 9 \\
Clinic history & $\mathrm{n}=24$ \\
$\quad$ Trauma & 12 \\
$\quad$ Significant abdominal surgery & 18 \\
No previous surgery & 4 \\
\hline Presenting complication & $\mathrm{n}=30$ \\
Small bowel obstruction & 20 \\
\hline Enterocutaneous fistula & 8 \\
\hline
\end{tabular}

blood vessels within mesenteric tissue [8]. The fibrous septa are mainly composed of fibroblasts, cellular fasciitis-like areas and trabeculae of osteoid filled with osteoblasts. Importantly the osteoblasts lack cytologic features consistent with malignancy [9]. The most important histologic feature that distinguishes HMO from extraskeletal osteosarcoma is the "zone phenomena." The "zone phenomena" is described as the immature central portion of the lesion which is typified by hypercellularity, atypia, and mitosis. This is surrounded by primitive osteoid progressing to mature osteoid with an outer zone of mature or lamellar bone [9]. This organized maturation of cells excludes the diagnosis of malignancy.

HMO remains a difficult preoperative diagnosis to make. However, if characteristic CT findings are present, a diagnosis of HMO can be made with confidence. Early features of HMO are usually evident 1 month from the initial injury and include linear and branching high-attenuation structures within the fat of the mesentery or omentum [10]. Just as important as making the diagnosis of HMO, is excluding other intra-abdominal pathologies, which are commonly found in the setting of pervious abdominal trauma or surgery. These include oral contrast leak from perforated viscus or anastomotic leak and active extravasation of intravascular contrast from bleeding. It is also critical to be able to differentiate HMO from dystrophic calcification within mesenteric neoplasm.

Differentiating HMO from extraluminal contrast is that extraluminal contrast tends to pool in dependent areas. In addition, if the contrast leak is due to a perforated viscus, the extraluminal contrast is often accompanied by pneumoperitoneum [10]. The key finding distinguishing an active bleed from $\mathrm{HMO}$ is the attenuation of the mass. A mass associated with hemoperitoneum is isoattenuating and HMO being hyperattenuating when compared to the contrast in the abdominal aorta [10]. Lastly, mesenteric neoplasm with dystrophic calcification within mesenteric neoplasm generally has a more punctate or 
amorphous appearance compared to the trabecular and cortical architecture of HMO.

A literature search revealed 44 cases of HMO. The results of the literature are summarized in Table 1 [1-23]. The vast majority of the cases occurred in males, with only two cases reported in females. There is a wide age distribution (12 - 80 years old), and most cases occurred in the fourth to sixth decade of life. Our patient, 24 years old, is within the age distribution, slightly younger than most patients who were found to have HMO. Nearly all cases of HMO occurred in the setting of previous abdominal surgery, including 12 with a history of trauma, 18 with a history of significant abdominal surgery and only four cases occurring in patients with no trauma or surgical history. The most common location for HMO was the mesentery, with 39 cases occurring in the mesentery and six reported in the omentum. In addition, nine cases reported concomitant finding of abdominal wall ossification.

Patients typically presented by 3 - 4 weeks from the initial operation. There was a wide range of presentation times - the earliest being 2 weeks and the latest being an incidental finding in an asymptomatic patient 7 years after the initial insult. Of the 30 cases that reported a complication upon presentation, there were 20 small bowel obstructions, eight enterocutaneous fistulas, one mass effect with pain, and one peritonitis. A small number $(n=4)$ of reports discussed the finding of elevated serum alkaline phosphatase in patients with HMO. Alkaline phosphatase is likely elevated as a result of osteoblastic activity and bone formation [5]. Of these, three reports noted a peak alkaline phosphatase level occurring around 3 weeks after the inciting event. Similarly, our patient had an elevated alkaline phosphatase of 215 at 3 weeks and 6 days following his initial surgery.

\section{Conclusion}

In summary, the case described here represents the ninth case of a patient with HMO presenting with an enterocutaneous fistula, adding to a growing body of literature of this rare consequence of abdominal surgery. The typical presentation of $\mathrm{HMO}$ is a young to middle-aged male with a small bowel obstruction or enterocutaneous fistula following major abdominal trauma or surgery. HMO is a difficult preoperative diagnosis to make; characteristic radiographic findings and an elevated alkaline phosphatase in setting of previous extensive abdominal trauma or surgery should raise the suspicion of this rare condition.

\section{References}

1. Ioannidis O, Sekouli A, Paraskevas G, Kotronis A, Chatzopoulos S, Papadimitriou N, Konstan-tara A, et al. Intra-abdominal heterotopic ossification of the peritoneum following traumatic splenic rupture. J Res Med Sci. 2012;17(1):92-95.

2. Hansen O, Sim F, Marton PF, Gruner OP. Heterotopic ossification of the intestinal mesentery. Report of a case following intraabdominal surgery. Pathol Res Pract. 1983;176(2-4):125-130.
3. Wilson JD, Montague CJ, Salcuni P, Bordi C, Rosai J. Heterotopic mesenteric ossification ('in-traabdominal myositis ossificans'): report of five cases. Am J Surg Pathol. 1999;23(12):1464-1470.

4. Como JJ, Yowler CJ, Malangoni MA. Extensive heterotopic mesenteric ossification after pene-trating abdominal trauma. J Trauma. 2008;65(6):1567.

5. Reynoso J, Christensen D, R L. Heterotopic mesentric ossifcation as a cause of per-sisten enterocutaneous fistula: overview of the literature and addition of a new case. European Suergery. 2012;44(4):285-290.

6. Myers MA, Minton JP. Heterotopic ossification within the small-bowel mesentery. Arch Surg. 1989;124(8):982983.

7. Obeid A, Sarhane K, Berjaoui T, Abiad F. Heterotopic intra-abdominal ossification in a com-plex ventral hernia defect. J Wound Care. 2014;23(2 Suppl):S5-9.

8. Zamolyi RQ, Souza P, Nascimento AG, Unni KK. Intraabdominal myositis ossificans: a report of 9 new cases. Int J Surg Pathol. 2006;14(1):37-41.

9. Androulaki A, Chatzoulis G, Kalahanis N, Lazaris AC. Heterotopic mesenteric ossification: a rare reactive process. J Gastroenterol Hepatol. 2005;20(4):664-666.

10. Baker JC, Menias CO, Bhalla S. Bone in the belly: traumatic heterotopic mesenteric ossifica-tion. Emerg Radiol. 2012;19(5):429-436.

11. Ackerman LV. Extra-osseous localized non-neoplastic bone and cartilage formation (so-called myositis ossificans): clinical and pathological confusion with malignant neoplasms. J Bone Joint Surg Am. 1958;40-A(2):279298.

12. Bovo G, Romano F, Perego E, Franciosi C, Buffa R, Uggeri F. Heterotopic mesenteric ossifica-tion ("intraabdominal myositis ossificans"): a case report. Int J Surg Pathol. 2004;12(4):407-409.

13. Hakim M, McCarthy EF. Heterotopic mesenteric ossification. AJR Am J Roentgenol. 2001;176(1):260-261.

14. Hashash JG, Zakhary L, Aoun EG, Refaat M. Heterotopic mesenteric ossification. Colorectal Dis. 2012;14(1):e2930 .

15. Hicks CW, Velopulos CG, Sacks JM. Mesenteric calcification following abdominal stab wound. Int J Surg Case Rep. 2014;5(8):476-479.

16. Kidambi S, Poll M, Kaplan FG, Shaker J. Heterotopic Ossification in the Mesentery After Abdominal Surgery. The Endocrinologist. 2006;16(6):323-328

17. Nabulyato WM, Alsahiem H, Hall NR, Malata CM. CASE REPORT An Unusual Case of Ab-dominal Compartment Syndrome Following Resection of Extensive Posttraumatic Mesenteric Ossi-fication. Eplasty. 2013;13:e13.

18. Patel RM, Weiss SW, Folpe AL. Heterotopic mesenteric ossification: a distinctive pseudosar-coma commonly associated with intestinal obstruction. Am J Surg Pathol. 2006;30(1):119-122.

19. Shi X, Zhang W, Nabieu PF, Zhao W, Fu C. Early postoperative heterotopic omental ossifica-tion: report of a case. Surg Today. 2011;41(1):137-140.

20. Tonino BA, van der Meulen HG, Kuijpers KC, Mallens WM, van Gils AP. Heterotropic mesen-teric ossification: 
a case report (2004:10b). Eur Radiol. 2005;15(1):195197.

21. Torgersen Z, Osmolak A, Bikhchandani J, Forse AR. Ectopic bone in the abdominal cavity: a surgical nightmare. J Gastrointest Surg. 2013;17(9):1708-1711.

22. Yannopoulos K, Katz S, Flesher L, Geller A, Ber- roya R. Mesenteritis ossificans. Am J Gastro-enterol. 1992;87(2):230-233.

23. Yushuva A, Nagda P, Suzuki K, Llaguna OH, Avgerinos D, Goodman E. Heterotopic mesenteric ossification following gastric bypass surgery: case series and review of literature. Obes Surg. 2010;20(9):1312-1315. 